\title{
Fluctuation contribution to the specific heat in non-Fermi models for superconductivity
}

\author{
I. Tifrea, I. Grosu and M. Crisan \\ Department of Theoretical Physics, University of Cluj, 3400 Cluj, Romania
}

\begin{abstract}
We investigate the fluctuation contribution to the specific heat of a twodimensional superconductor with a non-Fermi normal state described by a Anderson Green's function $G(\mathbf{k}, i \omega)=\omega_{c}^{-\alpha} /\left(i \omega-\varepsilon_{\mathbf{k}}\right)^{1-\alpha}$. The specific heat corrections contain a term proportional to $\left(T^{2 \alpha}-T_{c}^{2 \alpha}\right)^{-1}$ and another logarithmic one. We defined a coherence length as function of the non-Fermi parameter $\alpha$, which showed that a crossover study between BCS and Bose-Einstein condensation is possible by varying $\alpha$ in an interval $0 \div \alpha_{c r}$. By comparing our theoretical results with the experimental data for HTSC materials, we reobtained the value for $\alpha$, corresponding to such systems, of the order $0.3 \div 0.45$. We also reobtained the critical temperature for such a superconductor using the Thouless criterion.
\end{abstract}




\section{INTRODUCTION}

After the discovery of high critical temperature superconductors (HTSC) a great number of experimental works showed that the usual Fermi liquid description is unappropriated for these materials. As a consequence there are several phenomenological model 1213 proposed in order to explain the high critical temperature $T_{c}$ and the nonmetallic behavior of such materials. An important role in the mechanism of superconductivity is related to the fluctuation effects which are very important in the critical region. One of the parameter that characterize the normal state properties of a HTSC is the specific heat. Thompson and Kresin 1 , showed that in the absence of a magnetic field $\mathbf{B}=0$, in the critical region $\left(T \rightarrow T_{c}, T>T_{c}\right)$ the corrections in the specific heat obtained due to the fluctuation effects are proportional to $\left(T-T_{c}\right)^{-1 / 2}$. These corrections correspond to the classical region of fluctuation 1 .

Grosul reconsidered the problem for a two-dimensional superconductor with a logarithmic density of states and he showed that the corrections due to the fluctuation effects in the specific heat consist in two terms, one proportional to $\left(T-T_{c}\right)^{-1}$ and another logarithmic one.

In a previous paper 8 we derived the form for the specific heat jump in a non-Fermi superconductor described by the Anderson model. In our calculations we considered that the normal state Green's function has a similar form as the one from 1D interacting fermion systems 9 . A similar choice for the Green's function $G(\mathbf{k}, i \omega)=\omega_{c}^{-\alpha} /\left(i \omega-\varepsilon_{\mathbf{k}}\right)^{1-\alpha}$, where $\omega_{c}$ is a frequency cutoff introduced to makes the Green's function dimensionally correct and $\alpha$ is a parameter related to the anomalous Fermi surface, was made by Chakravarty and Anderson 10 in order to study the interlayer mechanism of the cuprate-oxides. The model has 
been developed by Sudbo11 who showed that the parameter $\alpha$ has to satisfy $0<\alpha<0.5$.

The aim of this work is to calculate the specific heat corrections of a two-dimensional non-Fermi system using the Anderson model. In Section II we derive the particle-particle polarization for the model and we reobtain the critical temperature $T_{c}$. We introduce using the fluctuation propagator a Ginzburg-Landau coherence length extrapolated to zero temperature, which in our calculations is function of the parameter $\alpha$. In Section III we evaluate the correction terms in the specific heat due to the fluctuation effects. In Section IV we analyze our results.

\section{PARTICLE-PARTICLE POLARIZATION}

In order to evaluate the corrections in the specific heat inside the critical region above $T_{c}$ first we have to calculate the Green's function connected with the Cooper pairs. It was showed by Schmide 3 that this Green's function is given by

$$
D\left(\mathbf{q}, \omega_{n}\right)=\left[\frac{1}{V}+\Pi(\mathbf{q}, \omega)\right]^{-1}
$$

where $V$ is the interaction strength and $\Pi\left(\mathbf{q}, \omega_{n}\right)$ is the usual particle-particle polarization given by

$$
\Pi\left(\mathbf{q}, \omega_{m}\right)=T \sum_{n} \int \frac{d^{2} k}{(2 \pi)^{2}} G\left(\mathbf{k}, \omega_{n}\right) G\left(\mathbf{q}-\mathbf{k}, \omega_{m}-\omega_{n}\right)
$$

with

$$
G\left(\mathbf{k}, \omega_{n}\right)=\frac{\omega_{c}^{-\alpha}}{\left(i \omega_{n}-\varepsilon_{\mathbf{k}}\right)^{1-\alpha}}
$$

The behavior of the Green function given by Eq. (3) for $\omega \rightarrow 0$ has been analyzed in Ref. 12 and the anomalous $\omega$-dependence of it in the interval $-\omega_{c}, \omega_{c}$ gives a softer singularity than 
1/ $\omega$ specific for the Green function of the Fermi liquid.

We remark that in the usual Fermi liquid theory $(\alpha=0)$, for a constant density of states, in a three dimensional system we reobtained the standard result (See Ref. 13)

$$
D\left(\mathbf{q}, \omega_{n}\right)=\frac{1}{N_{0}\left[\tau+a\left|\omega_{n}\right|+\xi^{2} q^{2}\right]}
$$

where $N_{0}$ is the density of states at the Fermi level, $\tau=\left(T-T_{c}\right) / T_{c}, a=\pi / 8 T_{c}$ and $\xi$ is the Ginzburg-Landau coherence length extrapolated to the zero temperature.

In order to calculate the particle-particle polarization we will rewrite Eq. (2) in the following form

$$
\Pi\left(\mathbf{q}, \omega_{m}\right)=\int \frac{d^{2} k}{(2 \pi)^{2}} S\left(\mathbf{k}, \mathbf{q}, \omega_{m}\right)
$$

where

$$
S\left(\mathbf{k}, \mathbf{q}, \omega_{m}\right)=T \sum_{n} \frac{\omega_{c}^{-2 \alpha}}{\left(i \omega_{n}-\varepsilon_{\mathbf{k}}\right)^{1-\alpha}\left(i \omega_{m}-i \omega_{n}-\varepsilon_{\mathbf{q}-\mathbf{k}}\right)^{1-\alpha}}
$$

The evaluation of the sum over the Matsubara frequencies in Eq. (6) is made using a contour integral which involves the Fermi-Dirac distribution function, with the specification that in this integral we have to deal with two branch cuts related to the complex function involved in the summation. Another observation which has to be made is the fact that a correct evaluation of this sum is possible only on the assumption that we work at low frequencies $\omega_{m}$ and small wavevectors $\mathbf{q}$. In this way we get

$$
\begin{aligned}
S\left(\mathbf{k}, \mathbf{q}, \omega_{m}\right) & =\frac{\omega_{c}^{-2 \alpha} \sin [\pi(1-\alpha)]}{\pi} \\
& \times\left\{-\left(2 \varepsilon_{\mathbf{k}}\right)^{2 \alpha-1} B(1-2 \alpha, \alpha)\right. \\
& +\frac{2 \Gamma(\alpha)}{\sqrt{\pi}} \sum_{m=0}^{\infty}(-1)^{m} \frac{\left(2 \varepsilon_{\mathbf{k}}\right)^{\alpha-1 / 2}}{[\beta(m+1)]^{\alpha-1 / 2}} K_{\alpha-1 / 2}\left[\varepsilon_{\mathbf{k}} \beta(m+1)\right]
\end{aligned}
$$




$$
\begin{aligned}
& +(\alpha-1)\left(v_{F} q \cos \theta-\omega_{m}\right)\left[-\left(2 \varepsilon_{\mathbf{k}}\right)^{2 \alpha-2} B(2-2 \alpha, \alpha-1)\right. \\
& \left.+\frac{\Gamma(\alpha-1)}{\sqrt{\pi}} \sum_{m=0}^{\infty}(-1)^{m} \frac{\left(2 \varepsilon_{\mathbf{k}}\right)^{\alpha-1 / 2}}{[\beta(m+1)]^{\alpha-3 / 2}} K_{\alpha-3 / 2}\left[\varepsilon_{\mathbf{k}} \beta(m+1)\right]\right] \\
& +\frac{(\alpha-1)^{2}\left(v_{F} q \cos \theta\right)^{2}}{2}\left[-\left(2 \varepsilon_{\mathbf{k}}\right)^{2 \alpha-3} B(3-2 \alpha, a-2)\right. \\
& +\frac{\Gamma(\alpha-2)}{\sqrt{\pi}} \sum_{m=0}^{\infty}(-1)^{m} \frac{\left(2 \varepsilon_{\mathbf{k}}\right)^{\alpha-1 / 2}}{[\beta(m+1)]^{\alpha-5 / 2}} K_{\alpha-3 / 2}\left[\varepsilon_{\mathbf{k}} \beta(m+1)\right] \\
& \left.+\frac{2 \Gamma(\alpha-1)}{\sqrt{\pi}} \sum_{m=0}^{\infty}(-1)^{m} \frac{\left(2 \varepsilon_{\mathbf{k}}\right)^{\alpha-3 / 2}}{[\beta(m+1)]^{\alpha-3 / 2}} K_{\alpha-3 / 2}\left[\varepsilon_{\mathbf{k}} \beta(m+1)\right]\right]
\end{aligned}
$$

where for the validity of this formula $\alpha$ should be between 0 and $0.5, B(x, y)$ is the beta function, $\Gamma(x)$ is the Euler's gamma function and $K_{\nu}(z)$ is the Bessel function of imaginary argument.

Using Eqs. (5) and (7) is very simple to evaluate the particle-particle polarization

$$
\begin{aligned}
\Pi(\mathbf{q}, \omega) & =\frac{2 N_{0} \sin [\pi(1-\alpha)]}{\pi} \\
& \times\left\{-\frac{2^{2 \alpha-1} B(1-2 \alpha, \alpha)}{2 \alpha}\left(\frac{\omega_{D}}{\omega_{c}}\right)^{2 \alpha}+\Gamma^{2}(\alpha) \frac{1-2^{1-2 \alpha}}{2^{1-2 \alpha}} \zeta(2 \alpha)\left(\frac{T}{\omega_{c}}\right)^{2 \alpha}\right. \\
& +\frac{i \omega(1-\alpha)}{\omega_{c}}\left[-\frac{2^{2 \alpha-2} B(2-2 \alpha, \alpha-1)}{2 \alpha-1}\left(\frac{\omega_{D}}{\omega_{c}}\right)^{2 \alpha-1}\right. \\
& \left.+\frac{\left.\Gamma(\alpha-1) \Gamma(\alpha-1 / 2) \frac{1-2^{2-2 \alpha}}{2^{2-2 \alpha}} \zeta(2 \alpha-1)\left(\frac{T}{\omega_{c}}\right)^{2 \alpha-1}\right]}{\sqrt{\pi}}\right] \\
& +\frac{\left(v_{F} q\right)^{2}(1-\alpha)^{2}}{4 \omega_{c}^{2}}\left[-\frac{2^{2 \alpha-3} B(3-2 \alpha, \alpha-2)}{2 \alpha-2}\left(\frac{\omega_{D}}{\omega_{c}}\right)^{2 \alpha-2}\right. \\
& \left.\left.+\left(\frac{2 \Gamma(\alpha-2) \Gamma(\alpha-1 / 2)}{\sqrt{\pi}}+\Gamma^{2}(\alpha-1)\right) \frac{1-2^{3-2 \alpha}}{2^{3-2 \alpha}} \zeta(2-2 \alpha)\left(\frac{T}{\omega_{c}}\right)^{2 \alpha-2}\right]\right\}
\end{aligned}
$$

where $\zeta(x)$ is the Riemann's zeta function.

In the case of zero frequency and zero wavevector $\Pi(\mathbf{q}=0, i \omega=0)$ we can obtain the critical temperature for normal state-superconductivity phase transition using the Thouless criterion

$$
1+\operatorname{VRe} \Pi(\mathbf{q}=0, i \omega=0)=0
$$


It is easy to see that we reobtained the previous result from Ref. 14, 15

$$
T_{c}^{2 \alpha}=\frac{1}{C(\alpha)}\left[D(\alpha) \omega_{D}^{2 \alpha}-\frac{\omega_{c}^{2 \alpha}}{A(\alpha) \lambda}\right]
$$

where $\lambda=N_{0} V, C(\alpha)=\Gamma^{2}(\alpha)\left(1-2^{1-2 \alpha}\right) \zeta(2 \alpha), A(\alpha)=2^{2 \alpha} \sin [\pi(1-\alpha)] / \pi$ and $D(\alpha)=$ $B(1-2 \alpha, \alpha) / 2 \alpha$.

Using Eqs. (8) and (10) the fluctuation propagator can be written in the following form

$$
\begin{aligned}
D^{-1}(\mathbf{q}, \omega) & \cong N_{0} A(\alpha) C(\alpha)\left[\left(\frac{T}{\omega_{c}}\right)^{2 \alpha}-\left(\frac{T_{c}}{\omega_{c}}\right)^{2 \alpha}\right] \\
& +N_{0} A(\alpha)\left[\frac{i \omega}{T} M(\alpha)(1-\alpha)+\frac{\left(v_{F} q\right)^{2}}{4 T^{2}} N(\alpha)(1-\alpha)^{2}\left(\frac{T_{c}}{\omega_{c}}\right)^{2}\right]
\end{aligned}
$$

where

$$
\begin{gathered}
M(\alpha)=-\frac{B(2-2 \alpha, \alpha-1)}{2(2 \alpha-1)}\left(\frac{\omega_{D}}{\omega_{c}}\right)^{2 \alpha-1} \frac{T_{c}}{\omega_{c}} \\
+\frac{\Gamma(\alpha-1) \Gamma(\alpha-1 / 2)}{2 \sqrt{\pi}}\left(1-2^{2-2 \alpha}\right) \zeta(2 \alpha-1)\left(\frac{T_{c}}{\omega_{c}}\right)^{2 \alpha} \\
N(\alpha)=-\frac{B(3-2 \alpha, \alpha-2)}{4(2 \alpha-2)}\left(\frac{\omega_{D}}{\omega_{c}}\right)^{2 \alpha-2} \\
+\left(\frac{2 \Gamma(\alpha-2) \Gamma(\alpha-1 / 2)}{\sqrt{\pi}}+\Gamma^{2}(\alpha-1)\right) \frac{1-2^{3-2 \alpha}}{4} \zeta(2-2 \alpha)\left(\frac{T_{c}}{\omega_{c}}\right)^{2 \alpha-2}
\end{gathered}
$$

which can be written also in the following form

$$
D(\mathbf{q}, \omega)=\frac{1}{N_{0}\left[b \tau+i a \omega+\xi_{0}^{2}(\alpha) q^{2}\right]}
$$

with $a=(1-\alpha) M(\alpha) A(\alpha) / T, b \cong A(\alpha) C(\alpha)\left(T_{c} / \omega_{c}\right)^{2}, \tau=\left(T / T_{c}\right)^{2 \alpha}-1$ and

$$
\xi_{0}^{2}(\alpha) \cong \frac{v_{F}^{2}(1-\alpha)^{2}}{4 \omega_{c}^{2}} N(\alpha) A(\alpha)
$$

If we compare Eqs. (14) and (4) we can define a Ginzburg-Landau coherence length extrapolated to zero temperature, which now is a function of the non-Fermi parameter $\alpha$. 
As it is known an important parameter related to the crossover theory between a BCS like formalism and a Bose-Einstein condensation formalism 16 is $k_{F} \xi_{0}$. In the BCS limit the value of such a parameter is about $10^{3} \div 10^{4}$ and in the Bose-Enstein condensation limit is zero. Experimental data showed that in the case of HTSC materials this parameter is between this two limits, for example in the case of $\mathrm{YBa}_{2} \mathrm{Cu}_{3} \mathrm{O}_{7-\delta}$ is about $5 \div 10$. In Fig. (1) we plot the variation of the parameter $k_{F} \xi_{0}$ (with the critical temperature corresponding to the optimal doping of $\left.\mathrm{YBa}_{2} \mathrm{Cu}_{3} \mathrm{O}_{7-\delta}\right)$ as a function of the non-Fermi parameter $\alpha$. The limit $\alpha \rightarrow 0$ correspond to the overdoped limit where the usual Fermi liquid theory still works for the normal state properties. The other limit $\alpha \rightarrow \alpha_{c r}$ correspond to the underdoped limit where the usual Fermi liquid theory breaks down. In this limit as we can see from Fig. (1) the coherence length is going to zero, which shows that here a Bose-Einstein condensation of strong coupling pairs $(\xi \rightarrow 0)$ is more close to the real picture of the system. The intermediate limit $(\alpha \rightarrow 10)$, which correspond to the optimal doping of $\mathrm{YBa}_{2} \mathrm{Cu}_{3} \mathrm{O}_{7-\delta}$, shows that the non-Fermi parameter $\alpha$ should be around $0.30 \div 0.45$, result which is in agreement with our previous calculation of the specific heat jump at the critical temperature 8 .

\section{FLUCTUATION CONTRIBUTION TO THE SPECIFIC HEAT}

The specific heat corrections due to the fluctuations around the critical temperature, will be calculated by having an additional term in the thermodynamic potential. Inside the critical region only the zero frequency term is important

$$
\Omega_{f l}=T \sum_{\mathbf{q}} \ln \left[D^{-1}(\mathbf{q}, i \omega=0)\right]
$$


Using the well known thermodynamic relations $S=-\partial \Omega_{f l} / \partial T$ and $C=T \partial S / \partial T$ the specific heat correction close to $T_{c}$ is

$$
C_{f l} \cong-T_{c} \frac{\partial^{2} \Omega_{f l}}{\partial \tau^{2}}\left(\frac{\partial \tau}{\partial T}\right)^{2}-T_{c} \frac{\partial \Omega_{f l}}{\partial \tau} \frac{\partial^{2} \tau}{\partial T^{2}}
$$

From Eqs. (14) and (17) we get

$$
C_{f l} \cong \frac{b \alpha^{2}}{\pi \xi_{0}^{2}(\alpha)} \frac{T_{c}^{2 \alpha}}{T^{2 \alpha}-T_{c}^{2 \alpha}}+\frac{b \alpha(1-2 \alpha)}{2 \pi \xi_{0}^{2}(\alpha)} \ln \left(\frac{2 m E_{F} \xi_{0}^{2}(\alpha)}{b} \frac{T_{c}^{2 \alpha}}{T^{2 \alpha}-T_{c}^{2 \alpha}}\right)
$$

The result contained in Eq. (18) is valid only in the critical region when we consider the classical fluctuations (region where the random phase approximation is still valid). Close to the transition point the interaction between electrons and Cooper pairs are very important, so we expect that in some way the electronic Green's function is affected. As a consequence there is a contribution $\delta \Pi$ to the particle-particle polarization $\Pi$ in Eq. (2), which can be written as

$$
\delta \Pi=2 T^{2} \sum_{n} \int \frac{d^{2} p}{(2 \pi)^{2}} G^{2}\left(\mathbf{k}, \omega_{n}\right) G^{2}\left(-\mathbf{k},-\omega_{n}\right) \int \frac{d^{2} q}{(2 \pi)^{2}} D(\mathbf{q}, i \omega=0)
$$

If we perform the integrals over $\mathbf{k}$ and $\mathbf{q}$, and if we sum over the Matsubara frequencies $\omega_{n}$ we get

$$
\delta \Pi \cong \frac{B(1 / 2,2(1-\alpha)-1 / 2)}{4 \pi^{3}} \frac{\omega_{c}^{-4 \alpha}}{\left(\pi T_{c}\right)^{1-4 \alpha}} \frac{2^{3-4 \alpha}-1}{2^{3-4 \alpha}} \zeta(3-4 \alpha) \ln \frac{2 m E_{F} \xi_{0}^{2}(\alpha)}{b \tau}
$$

With this correction the particle-particle polarization becomes $\Pi_{t}=\Pi+\delta \Pi$ and the fluctuation propagator can be written as

$$
D_{t}^{-1}=D^{-1}+\delta \Pi
$$

Using this definition very close to the transition temperature the significant contribution due to the fluctuations will be 


$$
\Omega_{t} \cong g \ln \left|c \ln \frac{2 m E_{F} \xi_{0}^{2}(\alpha)}{b \tau}\right|
$$

where $g$ is a constant which contains the critical temperature $T_{c}$ and a cutoff wavevector of order of $q_{F}$ (the Fermi wavevector) and $c$ is the constant in front of the logarithmic function in Eq. (2Q). With this thermodynamic potential the fluctuation contribution to the specific heat close to the critical temperature becomes

$$
C_{t} \cong \frac{4 \alpha^{2} g}{T_{c}}\left[\tau^{2} \ln \frac{2 m E_{F} \xi_{0}^{2}(\alpha)}{b \tau}\right]^{-1}+\frac{2 \alpha(2 \alpha-1) g}{T_{c}}\left[\tau \ln \frac{2 m E_{F} \xi_{0}^{2}(\alpha)}{b \tau}\right]^{-1}
$$

As we can see from Eqs. (23) and (18) the effect of the interaction between electrons and fluctuations should be very important, at least close enough to $T_{c}$. The only question is if the formula from Eq. (23) is valid in the framework of the performed approximations. There are several observations which make questionable this formula. The most important one is the fact that in Eq. (19) we used instead of a renormalized fluctuation propagator the bare one, which gives us a different behavior as a function of the wavevector $\mathbf{q}$ for $\delta \Pi$. In fact Eq. (19) should be a self consistent equation for $\delta \Pi$, which in our work is solved in the zero order.

\section{DISCUSSION}

We evaluated the contribution of the fluctuation contributions in the specific heat of a non-Fermi system described by the Anderson modell. We derived the form of the particleparticle polarization, and we showed that the critical temperature can be reobtained using the Thouless criterion. Using the fluctuation propagator $D(\mathbf{q}, i \omega)$ we introduced a coherence length $\xi_{0}(\alpha)$, which inn our calculations is function of the non-Fermi parameter $\alpha$. By varying 
$\alpha$ between zero and $\alpha_{c r}$ we showed in the case of $\mathrm{YBa}_{2} \mathrm{Cu}_{3} \mathrm{O}_{7-\delta}$ that the system can be described using the BCS theory or by the Bose-Einstein condensation of preformed pairs. In the case of optimal doping we reobtained for $\alpha$ the same values as in our previous paper $\alpha=0.3 \div 0.45$. As a consequence we consider that the numerical analysis in Ref. 14, 15 should be made on a scale $\alpha=0.3 \div 0.45$, not on the scale very close to the value $\alpha=0$.

In the third section of our paper we calculated the fluctuation contribution in the specific heat close to the critical temperature $T_{c}$, related to the classical fluctuation region, where the random phase approximation still remains valid. The result obtained by considering the fluctuation contributions give two different terms, the significant one being proportional to $\left(T^{2 \alpha}-T_{c}^{2 \alpha}\right)^{-1}$. A similar result can be obtained if we consider the critical field $H_{c 2}(\alpha, T)$ obtained by Muthukumar et al. 4 using the Ginzburg-Landau theory. Close to the critical temperature $T_{c}$, where interaction between electrons and Cooper pairs becomes very important we calculated the behavior of the specific heat by introducing a correction in the particle-particle polarization. The corrections obtained due to this additional term is very important, the divergence in Eq. (23) being more powerfull than the one in Eq. (18). All these results are valid in the approximation of the "box corrections" for the fluctuations propagator.

Finally we mention that the superconducting state developed in Ref. 12 using this nonFermi model presents some differences to the results from Ref. 11 because of the phase factor in the Green function. However, using the model proposed in Ref. 11 in the limit $\alpha \rightarrow 0$ it was showed 15 that the critical temperature is identical to the BCS expression. 


\section{REFERENCES}

${ }^{1}$ P. W. Anderson Science 256, 1526 (1992)

${ }^{2}$ C. M. Varma, P. B. Littlewood, S. Schmitt-Rink, E. Abrahams and A. E. Ruckenstein Phys. Rev. Lett. 63, 1996 (1989)

${ }^{3}$ A. J. Millis, H. Monien and D. Pines Phys. Rev. B42, 167 (1990)

${ }^{4}$ R. S. Thompson and V. Kresin Mod. Phys. Lett. B2, 1159 (1988)

${ }^{5}$ R. A. Ferrel and H. Schmidt Phy. Lett. A25, 544 (1967)

${ }^{6}$ K. Maki Prog. Theor. Phys. 40, 193 (1968)

${ }^{7}$ I. Grosu J. of Supercond. 10, 227 (1997)

${ }^{8}$ I. Tifrea and M. Crisan Eur. Phys. J. B4, 175 (1998)

${ }^{9}$ V. Meden and K. Schonhammer Phys. Rev. B46, 15753 (1992); J. Voit Phys. Rev. B47, 6740 (1993); M. Fabrizio and A. Parola Phys. Rev. Lett. 70, 226 (1993)

${ }^{10}$ S. Chakravarty and P. W. Anderson Phys. Rev. Lett. 72, 3859 (1994)

${ }^{11}$ A. Sudbo Phys. Rev. Lett. 74, 2575 (1995); A. Sudbo and J. M. Wheatley Phys. Rev. B52, 6200 (1995)

${ }^{12}$ L. Yin and S. Chakravarty Int. J. Mod. Phys. B10, 805 (1996)

${ }^{13}$ A. Schmid Z. Physik 231, 324 (1970)

${ }^{14}$ V. N. Muthukumar, D. Sa and M. Sardar Phys. Rev. B52, 9647 (1995)

${ }^{15}$ I. Grosu, I. Tifrea, M. Crisan and S. Yoksan Phys. Rev. B56, 8298 (1997) 
${ }^{16}$ M. Randeria in "Bose-Einstein Condensation" Ed. by A. Griffin et al., Cambridge University Press (1995) 


\section{FIGURES}

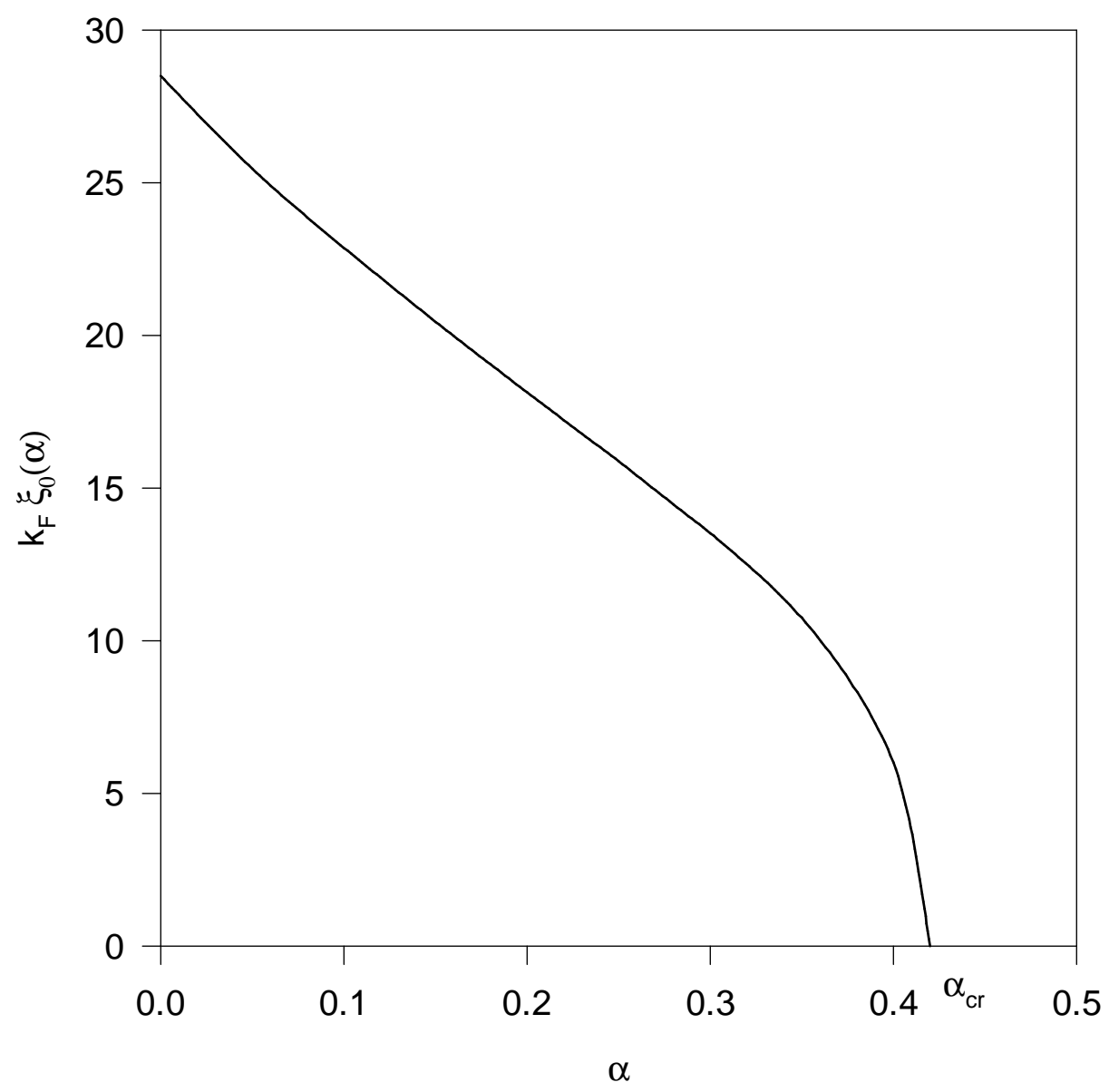

FIG. 1. The parameter $k_{F} \xi_{0}(\alpha)$ as a function of the non-Fermi parameter $\alpha\left(\omega_{D} / \omega_{c}=0.2\right.$, $\left.E_{c} / \omega_{c}=30, T_{c}=89 K\right)$ 